PROCEEDINGS OF THE

AMERICAN MATHEMATICAL SOCIETY

Volume 129, Number 5, Pages 1365-1366

S 0002-9939(00)05785-3

Article electronically published on October 11, 2000

\title{
HOLOMORPHIC PERTURBATION OF FOURIER COEFFICIENTS
}

\author{
THOMAS VILS PEDERSEN
}

(Communicated by Dale Alspach)

\begin{abstract}
Let $\mathbb{T}$ be the unit circle, let $\mathcal{B}$ be a Banach space continuously embedded in $L^{1}(\mathbb{T})$ and suppose that $\mathcal{B}$ is a Banach $L^{1}(\mathbb{T})$-module under convolution. We show that if $f(z)=\sum_{n=-\infty}^{\infty} a_{n} z^{n} \in \mathcal{B}$ and $F$ is holomorphic in a neighbourhood $U$ of 0 with $F(0)=0$ and $a_{n} \in U(n \in \mathbb{Z})$, then $\sum_{n=-\infty}^{\infty} F\left(a_{n}\right) z^{n} \in \mathcal{B}$.
\end{abstract}

Recently, Render ([3]) proved the result stated above for the case where $\mathcal{B}$ is the space $H^{\infty}(\mathbb{D})$ of bounded analytic functions on the open unit disc $\mathbb{D}$. In this note, we show that Render's result follows easily from the theory of so-called abstract Segal algebras, and that this approach allows us to generalize his result to a large class of spaces of functions. We also give a direct proof of this result.

For $f, g \in L^{1}(\mathbb{T})$, we define the convolution product $f * g$ by

$$
(f * g)\left(e^{i t}\right)=\frac{1}{2 \pi} \int_{\mathbb{T}} f\left(e^{i s}\right) g\left(e^{i(t-s)}\right) d s \quad\left(e^{i t} \in \mathbb{T}\right) .
$$

Then $\widehat{f * g}(n)=\widehat{f}(n) \widehat{g}(n)$, where $\widehat{f}(n)(n \in \mathbb{Z})$ are the Fourier coefficients of $f$.

Proposition 1. Let $\mathcal{B}$ be a Banach space continuously embedded in $L^{1}(\mathbb{T})$ and suppose that $\mathcal{B}$ is a Banach $L^{1}(\mathbb{T})$-module under convolution. Let $E=\{n \in \mathbb{Z}$ : there exists $f \in \mathcal{B}$ with $\widehat{f}(n) \neq 0\}$. Considered as a Banach algebra under convolution, every character on $\mathcal{B}$ is of the form

$$
f \mapsto \widehat{f}(n) \quad(f \in \mathcal{B})
$$

for some $n \in E$.

Proof. Let $L_{E}^{1}(\mathbb{T})=\left\{f \in L^{1}(\mathbb{T}): \widehat{f}(n)=0\right.$ for $\left.n \in \mathbb{Z} \backslash E\right\}$. For $n \in E$, choose $f \in \mathcal{B}$ with $\widehat{f}(n) \neq 0$. Then $e^{i n t}=\left(e^{i n t} * f\right) / \widehat{f}(n) \in \mathcal{B}$, so $\mathcal{B}$ contains every trigonometric polynomial in $L_{E}^{1}(\mathbb{T})$, and in particular $\mathcal{B}$ is dense in $L_{E}^{1}(\mathbb{T})$. Hence $\mathcal{B}$ is an abstract Segal algebra with respect to $L_{E}^{1}(\mathbb{T})$ in the sense of [1] Definition 1.1], so the result follows from [1, Theorem 2.1] (see [2, Theorem 6.2.4] for the case $E=\mathbb{Z}$ ).

Remark 2. Let $\mathcal{B}$ be a Banach space continuously embedded in $L^{1}(\mathbb{T})$ and for $f \in \mathcal{B}$ and $e^{i s} \in \mathbb{T}$, let $f_{s}\left(e^{i t}\right)=f\left(e^{i(t-s)}\right)\left(e^{i t} \in \mathbb{T}\right)$. If $f_{s} \in \mathcal{B}$ with $\left\|f_{s}\right\|_{\mathcal{B}}=\|f\|_{\mathcal{B}}$ and

Received by the editors July 20, 1999.

2000 Mathematics Subject Classification. Primary 42A16; Secondary 46J20.

This work was carried out at Université Bordeaux 1 while the author was holding a TMR Marie Curie postdoctoral grant from the European Commission.

(C)2000 American Mathematical Society 
$f_{s} \rightarrow f$ in $\mathcal{B}$ as $e^{i s} \rightarrow 1$, or if $\mathcal{B}$ is a dual space (like $H^{\infty}(\mathbb{D})$ ) and $f_{s} \rightarrow f$ weak star in $\mathcal{B}$ as $e^{i s} \rightarrow 1$, then

$$
g * f=\frac{1}{2 \pi} \int_{\mathbb{T}} g\left(e^{i s}\right) f_{s} d s \in \mathcal{B}
$$

and $\|g * f\|_{\mathcal{B}} \leq\|g\|_{L^{1}(\mathbb{T})}\|f\|_{\mathcal{B}}$ for $g \in L^{1}(\mathbb{T})$, so $\mathcal{B}$ is a Banach $L^{1}(\mathbb{T})$-module under convolution. This provides a large class of spaces of functions to which our results apply.

With $\mathcal{B}$ as above, let $\mathcal{A}=\mathcal{B} \oplus \mathbb{C} \delta_{1}$ be the Banach algebra obtained by adjoining the identity $\delta_{1}$ (the Dirac measure at $z=1$ ) to $\mathcal{B}$.

Corollary 3. For $f(z)=\sum_{n=-\infty}^{\infty} a_{n} z^{n} \in \mathcal{B}$, we have $\sigma_{\mathcal{A}}(f)=\left\{a_{n}: n \in E\right\} \cup\{0\}$.

Our main result now follows from the holomorphic functional calculus for Banach algebras (see, for instance, [4, Theorem 10.2.7]).

Theorem 4. Let $f(z)=\sum_{n=-\infty}^{\infty} a_{n} z^{n} \in \mathcal{B}$ and let $F$ be holomorphic in a neighbourhood $U$ of 0 with $F(0)=0$ and $a_{n} \in U(n \in \mathbb{Z})$. Then $\sum_{n=-\infty}^{\infty} F\left(a_{n}\right) z^{n} \in \mathcal{B}$.

Remark 5. Writing $F(z)=z G(z)(z \in U)$, the conclusion of the theorem takes the form $\sum_{n=-\infty}^{\infty} G\left(a_{n}\right) a_{n} z^{n} \in \mathcal{B}$; that is, the sequence $\left(G\left(a_{n}\right)\right)$ acts as a "local coefficient multiplier" on $f$.

We wish to point out that it is possible to give a proof of Corollary 3 (and thus of Theorem (4) which does not depend on the theory of abstract Segal algebras: Let $f(z)=\sum_{n=-\infty}^{\infty} a_{n} z^{n} \in \mathcal{B}$ and suppose that $\lambda \notin\left\{a_{n}: n \in E\right\} \cup\{0\}$. It is well known that every character on $L^{1}(\mathbb{T})$ is of the form $g \mapsto \widehat{g}(n)\left(g \in L^{1}(\mathbb{T})\right)$ for some $n \in \mathbb{Z}$, so it follows that $\lambda \notin \sigma_{L^{1}(\mathbb{T}) \oplus \mathbb{C} \delta_{1}}(f)$. Since the Fourier series of $\delta_{1}$ is $\sum_{n=-\infty}^{\infty} z^{n}$, we thus have

$$
\left(\lambda \delta_{1}-f\right)^{-1}=\sum_{n=-\infty}^{\infty} \frac{1}{\lambda-a_{n}} z^{n}=\frac{1}{\lambda} \sum_{n=-\infty}^{\infty} \frac{a_{n}}{\lambda-a_{n}} z^{n}+\frac{1}{\lambda} \delta_{1} \in L^{1}(\mathbb{T}) \oplus \mathbb{C} \delta_{1} .
$$

Moreover, since $\mathcal{B}$ is a Banach $L^{1}(\mathbb{T})$-module under convolution, we have

$$
\sum_{n=-\infty}^{\infty} \frac{a_{n}}{\lambda-a_{n}} z^{n}=\left(\lambda \delta_{1}-f\right)^{-1} * f \in \mathcal{B}
$$

so $\left(\lambda \delta_{1}-f\right)^{-1} \in \mathcal{A}$, and we deduce that $\lambda \notin \sigma_{\mathcal{A}}(f)$.

\section{REFERENCES}

[1] J.T. Burnham, Closed ideals in subalgebras of Banach algebras, Proc. Amer. Math. Soc. 32 (1972), 551-555. MR 45:4146

[2] H. Reiter, Classical harmonic analysis and locally compact groups, Oxford University Press, London, 1968. MR 46:5933

[3] H. Render, The maximal ideal space of $H^{\infty}(\mathbb{D})$ with respect to the Hadamard product, Proc. Amer. Math. Soc. 127 (1999), 1409-1411. MR 99h:46101

[4] W. Rudin, Functional analysis, McGraw-Hill Book Company, New York, 1973. MR 51:1315

Laboratoire de Mathématiques Pures, Université Bordeaux 1, 351, Cours de la LibéRation, F-33405 Talence CÉdex, France

E-mail address: vils@math.u-bordeaux.fr 